\title{
Mg-Isotopie in Magnesiten - eine Pilotstudie für den Lagerstätten-Isotopenkatalog Österreichs
}

\author{
Fritz Ebner ${ }^{1,3}$, Dorothee Hippler ${ }^{2}$, Martin Dietzel² und Heinrich Mali ${ }^{1}$ \\ 'Department Angewandte Geowissenschaften und Geophysik, Montanuniversität Leoben, Leoben, Österreich \\ ${ }^{2}$ Institut für Angewandte Geowissenschaften, Technische Universität Graz, Graz, Österreich \\ ${ }^{3} \mathrm{Graz}$, Österreich
}

Eingegangen 13. Dezember 2018; angenommen 15. Dezember 2018; online publiziert 10. Januar 2019

\begin{abstract}
Zusammenfassung: Zur systematischen Erfassung von Isotopendaten österreichischer Lagerstätten wurden erstmals stabile Mg-Isostope ( $\delta^{26} \mathrm{Mg}$ DSM-3) ausgewählter ostalpiner Spatmagnesitlagerstätten/-vorkommen (Hohentauern/ Sunk, Hochfilzen, Breitenau, Radenthein/Millstätter Alpe, Kaswassergraben) und des kryptokristallinen Kraubath Magnesits bestimmt. Zusätzlich wurde aus Vergleichsgründen und zur Charakterisierung wichtiger Typen von Magnesitlagerstätten auch ausländisches Material (Bela Stena/ Serbien, Bushveld/Südadfrika, Poldasht/NW Iran) untersucht. Dabei wurden für die global wichtigen Typen von Magnesitlagerstätten signifikat unterschiedliche $\delta^{26} \mathrm{MgDSM}_{\mathrm{D}-3^{-}}$ Werte ermittelt: Kraubath-Typ $-2.88 \%$ o bis $-1.80 \%$, VeitschTyp -1.54 bis $-0.49 \%$, Bela Stena-Typ -1.07 bis $-0.42 \%$, Poldasht-Typ -1.04 bis $+1.01 \%$. Diese lassen sich weitestgehend auf die unterschiedlichen Bildungsprozesse (z.B. Evaporation, Metasomatose) zurückführen.
\end{abstract}

Schlüsselwörter: Magnesitlagerstätten, Mg-Isotope, Spatmagnesit, Kryptokristalliner Magnesit, LagerstättenTypisierung, Österreich

Mg-isotopes in Magnesite-a Pilot Study for the Austrian Deposit's Isotope Database

Abstract: Magnesite of selected Austrian deposits/occurrences (sparry magnesite of Hohentauern/Sunk, Hochfilzen, Breitenau, Radenthein/Millstätter Alpe, Kaswassergraben and cryptocrystalline magnesite of Kraubath) were analysed for their stable $\mathrm{Mg}$-isotope composition ( $\delta^{26} \mathrm{MgDSM}-3$ ). This pilot study focussed on the extension/completion of isotope data of Austrian mineral deposits. Additionally, some foreign types of magnesite (Bela Stena/Serbia, Bushveld/South Africa, Poldasht/NW Iran) were also analysed for comparison in order to evaluate site-specific

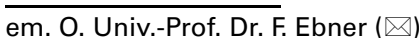

Waldteufelweg 13a,

$8047 \mathrm{Graz}$, Österreich

fritz.ebner@gmx.net characteristics. We found that the most important types of magnesite yield specific $\delta^{26} \mathrm{MgDSM}_{\mathrm{D}}-\mathrm{m}$-values: Kraubathtype $-2.88 \%$ up to $-1.80 \%$, Veitsch-type -1.54 up to $-0.49 \%$, Bela Stena-type-1.07 up to $-0.42 \%$ and Poldasht-type -1.04 up to $+1.01 \%$, the latter deciphering the different formation mechanisms.

Keywords: Magnesite deposits, Mg-isotopes, Sparry magnesite, Cryptocrystalline magnesite, Typification of deposits, Austria

\section{Einleitung}

In der Programmbeschreibung der GBA Forschungspartnerschaft: „Grundlagenorientierte Mineralrohstoffforschung" wird die systematische Erfassung von Isotopendaten österreichischer Lagerstätten als prioritär ausgewiesen, nachdem schon 2009 die Struktur einer derartigen Datenbank vorgestellt wurde [1]. Bedingt durch die damals verfügbaren Daten wurden in diesem Vorschlag aber nur die Isotope des O, C, H und S berücksichtigt. Das vorliegende Pilotprojekt zielt daher auf die stabile Mg-Isotopensignatur ( $\delta^{26} \mathrm{Mg}_{\mathrm{DSM}-3}$ ) aus Magnesiten (1) zur Ergänzung dieser Isotopendatenbank, (2) zur Charakterisierung von Magnesitlagerstätten und (3) zur Unterstützung für genetische Fragestellungen ab. Diesen Untersuchungen kommt ein innovativer Charakter zu, da bei der Projektplanung weltweit noch keine Angaben über Mg-Isotope in Magnesiten vorlagen und erst mit Projektbeginn (Sommer 2016) aus chinesischen Lagerstätten weltweit die ersten $\delta$-Mg Isotopenwerte aus Magnesiten publiziert wurden [2].

\section{Methodik}

Nach makroskopischer Materialauswahl wurden an polierten Gesteinsanschliffen nach $\mathrm{HCl}$-Ätzung und Auflichtmikroskopie möglichst homogene Bereiche definiert, aus 
denen über Mikrodrilling das Analysenmaterial gewonnen wurde. Aliquote von ca. $1 \mathrm{mg}$ wurden anschließend mit konzentrierter $\mathrm{HNO}_{3}$ aufgelöst und das $\mathrm{Mg}$ mittels einer zweistufigen lonenchromatographie chemisch abgetrennt [3]. Die Messung der Mg-Isotope erfolgte mit hochauflösender Multikollektor-Massenspektroskopie mit induktiv gekoppeltem Plasma (HR-MC-ICP-MS, Nu Instruments, Wexham, Großbritannien) im NAWI Zentrallabor für Wasser, Gesteine und Minerale der TU Graz. Die Mg-Isotopie wird in der $\delta$-Notation $\left(\delta^{25} \mathrm{Mg}\right.$ und $\left.\delta^{26} \mathrm{Mg}\right)$ als relative Abweichung zum Referenzmaterial DSM-3 in\%o angegeben. Ein $\delta$-Wert entspricht dabei mindestens vier Wiederholungsmessungen, mit einer analytischen Unsicherheit auf $\delta^{25} \mathrm{Mg}$ und $\delta^{26} \mathrm{Mg}$ von $\leq 0,10$ bzw. $0,15 \%$.

\section{Herkunft und geologische Charakteristik des Probenmaterials}

Unter der Prämisse dass Magnesite unterschiedlicher Genese und geologischer Situierung eine unterschiedliche Mg-Isotopie aufweisen, wurden aus Österreich folgende, verschiedenen metallogenetischen Bezirken [4, 5] angehörige Magnesite untersucht (Abb. 1):

1. Metasomatischer Spatmagnesit (Veitsch-Typ) der Lagerstätten Hohentauern/Sunk, Hochfilzen, Breitenau und Radenthein,

2. Grobkristalliner Spat-( „,Salinar-“)magnesit des Kaswassergrabens,

3. Kryptokristalliner Magnesit aus dem Serpentinit von Kraubath (Kraubath-Typ).

Tab. 1 zeigt die $\delta^{26} \mathrm{Mg}_{\mathrm{DSM}-3}$-Werte und anderen für die Isotopendatenbank relevanten Daten der untersuchten österreichischen Proben. Zur Absteckung der Variation der

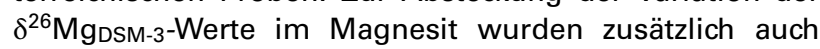
ausländische Lokalitäten mit zu österreichischen Lagerstätten/Vorkommen konträrem Magnesit-Bildungsmilieu untersucht (Tab. 2):
1. Sedimentärer kryptokristalliner Magnesit aus Bela Stena/Serbien,

2. Kryptokristalliner Magnesit aus dem Bushveld Komplex/ Südafrika,

3. Rezenter kryptokristalliner Magnesit aus Playas von Poldasht/NW Iran.

Bei Untersuchung im Rasterelektronenmikroskop zeigt sich, dass weder beim Spatmagnesit in Karbonatgesteinen noch beim Kraubath-Typ Magnesit in Serpentiniten mit monomineralischen Proben zu rechnen ist. Im Spatmagnesit finden sich neben Resten des nicht umgewandelten Dolomits immer mehrere Generationen von syn- bis postmagnesitischem Dolomit (Abb. 2). Der Magnesit vom Kraubath-Typ führt reichlich Reste von nicht umgewandeltem Serpentin (Abb. 3). Die feinstkörnigen chemisch gefällten Magnesite vom Bela Stena- und Poldasht-Typ sind dagegen homogen und nur durch klastisch eingetragene Sedimentkomponenten verunreinigt.

\subsection{Hohentauern/Sunk}

Die Lagerstätte ist an karbone geringmetamorphe Karbonatgesteine gebunden. Bevorzugt tritt grobkörniger Pinolit- und Bändermagnesit auf, für den ein metasomatisches Bildungsmodell und durch $\mathrm{Sm} / \mathrm{Nd}$ Datierungen ein oberkarbones bis unterpermisches Bildungsalter belegt ist. Dolomit in der Umhüllung des Magnesits, wie auch im Magnesit, entstammt unterschiedlichen Generationen [6].

Proben:

- Magnesit: H8, H8F, H 11 (Pinolitmagnesit), H9 (Bändermagnesit)

- Dolomit (H7-1 fossilführend, H7-2 grobkörnig) vom metasomatischen Kontakt zum Spatmagnesit (H7-3M, H73MD)

- Kokardendolomit: Dolomitlage der Kokarde (H14-1D), laminierte Dolomitkluft (H14-2D).
Abb. 1: Die Magnesitbezirke Österreichs mit Hinweis (Fettdruck) auf die untersuchten Lagerstätten/Vorkommen. Die Reichweite der in Magnesit und Dolomit gemessenen $\delta^{26} \mathrm{Mg}$-Werte ist ebenfalls angeführt. Abkürzungen: $M$ für Magnesit, D für Dolomit und KKD für Kokardendolomit
M A G N E S I T - B E Z I R K E

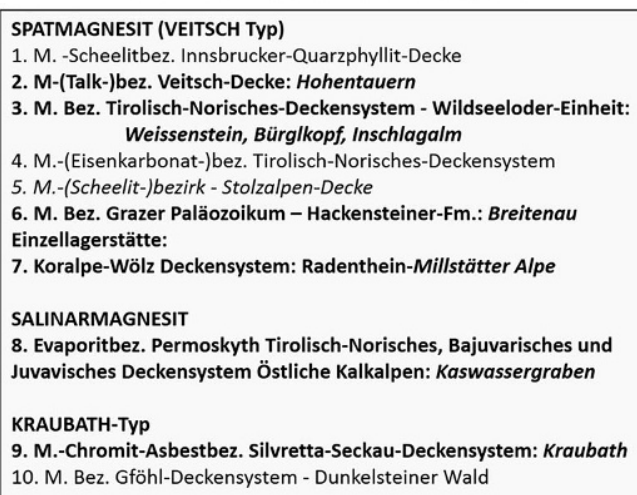
Weissenstein, Bürglkopf, Inschlagalm

4. M.-(Eisenkarbonat-)bez. Tirolisch-Norisches-Deckensystem

5. M.-(Scheelit-)bezirk - Stolzalpen-Decke

6. M. Bez. Grazer Paläozoikum - Hackensteiner-Fm.: Breitenau

Einzellagerstätte:

7. Koralpe-Wölz Deckensystem: Radenthein-Millstätter Alpe

SALINARMAGNESIT

8. Evaporitbez. Permoskyth Tirolisch-Norisches, Bajuvarisches und Juvavisches Deckensystem Östliche Kalkalpen: Kaswassergraben

KRAUBATH-Typ

9. M.-Chromit-Asbestbez. Silvretta-Seckau-Deckensystem: Kraubath

10. M. Bez. Gföhl-Deckensystem - Dunkelsteiner Wald

Untersuchte Lagerstätten

\begin{tabular}{|c|c|}
\hline $\begin{array}{l}\text { In Karbonatgesteinen } \\
\text { Veitsch-Typ }\end{array}$ & $\delta^{26} M g_{D S M-3}$ \\
\hline $\begin{array}{l}\text { 2. Hohentauern } \\
\qquad D:-0,58 \text { bis }-0,45\end{array}$ & $\begin{array}{r}\text { M: }-0.88 \text { bis }-0.62 \% \text { o } \\
\text { KKD: }-0.65 \text { bis }-0.75 \% \text { o }\end{array}$ \\
\hline 3. Hochfilzen & $\begin{array}{l}\text { M: }-1.54 \text { bis }-1.04 \% o \\
D:-1.22 \text { bis }-0.97 \% o\end{array}$ \\
\hline 6. Breitenau & $\begin{array}{l}\text { M: }-0.93 \text { bis }-0.72 \% \\
D:-0.13 \text { bis }+0,5 \%\end{array}$ \\
\hline 7. Radenthein & $\begin{array}{l}\text { M: }-0.84 \text { bis }-0.49 \% \text { o } \\
\text { D: }-2.02 \text { bis }-1.21 \% \text { o }\end{array}$ \\
\hline Salinarmagnesit & \\
\hline $\begin{array}{l}\text { 8. Kaswassergraben } \\
\text { In Ultrabasiten } \\
\text { Kraubath-Typ }\end{array}$ & $\begin{array}{l}\text { M: }-1,37 \text { bis }-1,27 \% \\
\text { D: -- }\end{array}$ \\
\hline 9. Kraubath & M: $-2,88$ bis $-1,93 \%$ o \\
\hline
\end{tabular}


Abb. 2: Metasomatisch gebildeter Spatmagnesit zeigt im Rasterelektronenmikroskop immer Kontaminationen durch Dolomit. (a Hohentauern, $\mathrm{H} 8$ ) postmagnesitisch gebildeter Dolomit (D) im hellgrauen Spatmagnesit (M) hellgrauen Spatmagnesit (M)
(b Breitenau, P13) Phantome von nicht umgewandeltem Dolomit (D) in grauem Spatmagnesit (M). (c Breitenau, P13) typischer Spatmagnesit
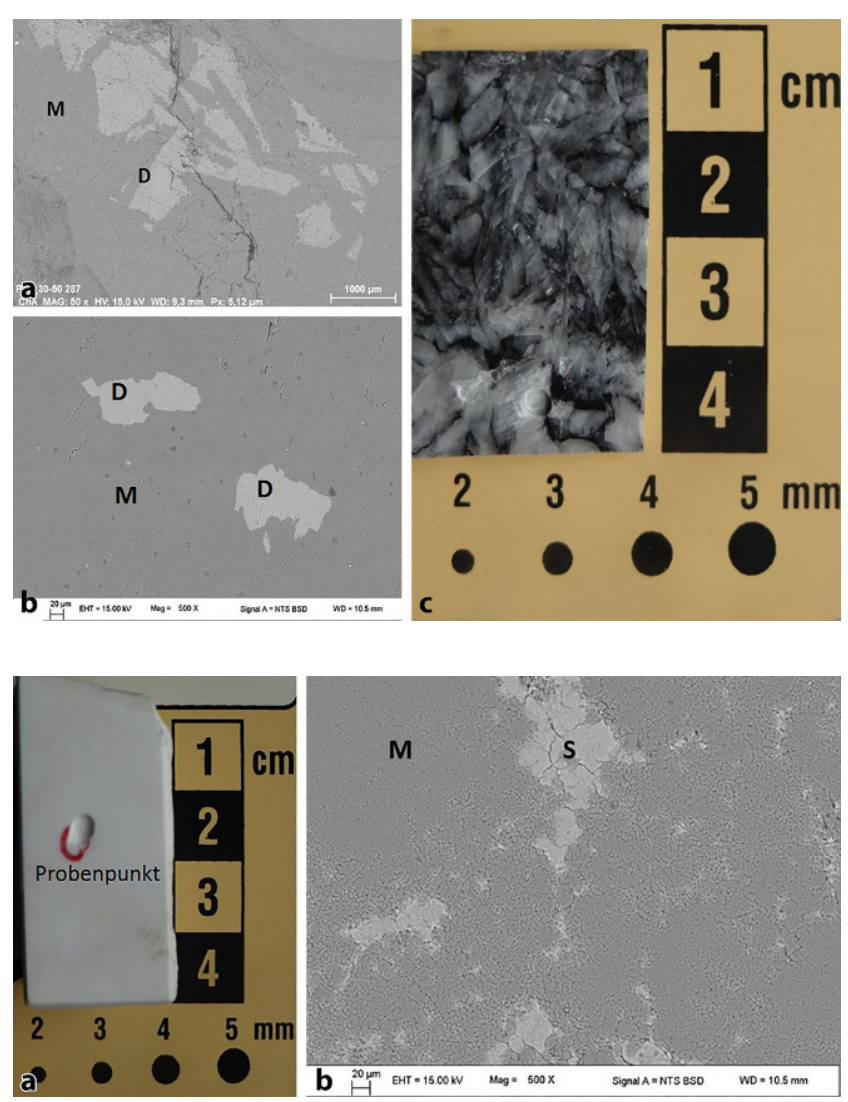

Abb. 3: a Selbst makroskopisch homogen erscheinender Kraubath-Magnesit (Au45) zeigt im Rasterelektronenmikroskop (b) intensive Verwachsungen von nicht umgewandeltem Serpentin (S) im kryprokristallinen Magnesit (M)

\subsection{Hochfilzen}

Im Bereich Weissenstein-Bürglkopf liegt das Magnesitlager in bis zu $500 \mathrm{~m}$ mächtigem Dolomit (Obersilur - Unterdevon), dem pelagische Kalke vorausgegangen sind. Die Primärgefüge sind durch die metasomatische Magnesitbildung weitgehend verdrängt. Der unterschiedlich gefärbte Magnesit ist meist feinkörnig. Am Bürglkopf findet sich auch eine feinkörnige braune, "sandig" anwitternde Varietät und auf der Inschlagalm ist zusätzlich typischer Spatmagnesit entwickelt $[7,8]$.

Proben:

- Dolomit Weissenstein: hof-1, hof-2

- Magnesit Bürgl: hof-9 (rot), hof-10 (hellgrau); Kontakt Dolomit (hof-11D) zu Magnesit (sandig, braun; hof-11M).

- Magnesit Inschlagalm: hof-12M, Dolomit des Kontaktes (hof-12D).

\subsection{Breitenau/Grazer Paläozoikum}

Der grobkörnige Magnesit (darunter auch Bänder- und Pinolitmagnesit) ist an graue bis $200 \mathrm{~m}$ mächtige unterdevone Dolomite gebunden. Späte Dolomitbildungen sind Rosszahndolomit, Redolomitisierung entlang von Spaltrissen/ Kristallgrenzen sowie pigmentreiche grobe Dolomitkristalle. Geochemie und Kontakte des Magnesits zum Wirtsgestein deuten auf eine metasomatische Magnesitbildung, die nach $\mathrm{Sm} / \mathrm{Nd}$-Datierungen in der Obertrias stattfand [9, 10].

Proben:

- Magnesit: P-Br1 (Magnesitsonne), P3 (grobkristallin), P13 (Pinolitmagnesit), P16M (Pinolitmagnesit in Dolomit P16D).

- Dolomit: P22a (grau), P16D (schwarz; darin aufsprossender Magnesit P16M)

\subsection{Radenthein/Millstätter Alpe}

Der 30-70 m mächtige Spatmagnesitkörper wird von Calcit- und Dolomitmarmoren begleitet, die zusammen in kretazisch geprägte Granatglimmerschiefer eingelagert sind. Vorherrschend ist neben grobkörnigem Pinolit- und Bändermagnesit auch weißer Spatmagnesit (Magnesitmarmor). Für ihre Genese werden metasomatische Modelle neben der Bildung in einem abgeschnürten Evaporitbecken angedacht $[4,11]$.

Proben:

- Spatmagnesit: R4-3, R5-2.1, R5-2.2, R5-2.3; Dolomit: R4-1, R5-1 


\begin{tabular}{|c|c|c|c|c|c|c|c|c|c|}
\hline & \multirow[t]{2}{*}{ Probe-Lokalität } & \multicolumn{2}{|c|}{ Koord. Gauss Krüger } & \multirow[t]{2}{*}{ Min } & \multirow[t]{2}{*}{ RG } & \multirow{2}{*}{$\begin{array}{l}\delta 25 \mathrm{Mg} \\
\mathrm{DSM}-3\end{array}$} & \multirow{2}{*}{$\begin{array}{l}\delta 26 \mathrm{Mg} \\
\mathrm{DSM}-3\end{array}$} & \multirow{2}{*}{$\begin{array}{l}\delta 180 \\
\text { VPDB }\end{array}$} & \multirow{2}{*}{$\begin{array}{l}\delta 13 C \\
\text { VPDP }\end{array}$} \\
\hline & & $\mathrm{x}$ & y & & & & & & \\
\hline \multicolumn{10}{|c|}{ HOHENTAUERN/SUNK: Magnesitbezirk Veitscher Decke } \\
\hline H7-1 & re Bachseite & 5257426 & 85515 & $\mathrm{D}$ & W & -0.34 & -0.58 & -16.18 & 0.21 \\
\hline $\mathrm{H} 7-2$ & re Bachseite & 5257426 & 85515 & $\mathrm{D}$ & $\mathrm{W}$ & -0.28 & -0.45 & -15.75 & 0.74 \\
\hline $\mathrm{H} 7-3 \mathrm{M}$ & li Bachseite & 5257439 & 85323 & SM & 1 & -0.32 & -0.64 & - & - \\
\hline H7-3MD & li Bachseite & 5257439 & 85323 & SM & 1 & -0.48 & -0.88 & -17.82 & 0.36 \\
\hline H7-3MD & li Bachseite & 5257439 & 85323 & $\mathrm{SM}$ & 1 & -0.48 & -0.88 & -17.82 & 0.36 \\
\hline $\mathrm{H} 8$ & re Bachseite & 5257426 & 85515 & SM & I & -0.34 & -0.62 & -19.09 & 0.21 \\
\hline $\mathrm{H} 8 \mathrm{~F}$ & re Bachseite & 5257426 & 85515 & $\mathrm{SM}$ & 1 & -0.34 & -0.64 & -18.72 & 0.25 \\
\hline $\mathrm{H} 9$ & re Bachseite & 5257426 & 85515 & $\mathrm{SM}$ & 1 & -0.30 & -0.68 & -18.75 & 0.30 \\
\hline $\mathrm{H} 11$ & re Bachseite & 5257426 & 85515 & SM & I & -0.36 & -0.63 & -19.42 & 0.10 \\
\hline H14-1D & re Bachseite & 5257426 & 85515 & $\mathrm{D}$ & KD & -0.43 & -0.75 & -14.41 & -0.15 \\
\hline H14-2D & re Bachseite & 5257426 & 85515 & $\mathrm{D}$ & $\mathrm{KD}$ & -0.37 & -0.65 & -13.82 & 0.78 \\
\hline \multicolumn{10}{|c|}{ HOCHFILZEN: Magnesitbez. Tir.-Nor. Deckensyt. Wildseeloder Einheit } \\
\hline hof-01 & Weissenstein Etage 11 & 5254410 & -57936 & $\mathrm{D}$ & W & -0.67 & -1.22 & -6.07 & 0.28 \\
\hline hof-02 & Weissenstein Etage 5 & 5254390 & -57936 & $\mathrm{D}$ & W & -0.38 & -0.67 & -9.67 & 8.32 \\
\hline hof-9 & Bürgl Sohle 3 & 5254624 & -55721 & SM & 1 & -0.66 & -1.26 & -9.68 & 0.54 \\
\hline hof-10 & Bürgl Sohle 1 & 5254619 & -55495 & SM & 1 & -0.55 & -1.04 & -10.51 & 1.36 \\
\hline hof-11M & Bürgl/Sohle 1 Bohrung & 5254605 & -55495 & SM & 1 & 0.62 & -1.21 & -12.77 & 1.32 \\
\hline hof-11D & Bürgl/Sohle 1 Bohrung & 5254605 & -55495 & $\mathrm{D}$ & W & -0.69 & -1.28 & -10.84 & 2.12 \\
\hline hof-12M & Inschlagalm Tagebau & 5254440 & -50370 & SM & 1 & -0.81 & -1.54 & -10.55 & 0.38 \\
\hline hof-12D & Inschlagalm Tagebau & 5254440 & -50370 & $\mathrm{D}$ & W & -0.54 & -0.97 & -12.00 & 0.30 \\
\hline \multicolumn{10}{|c|}{ BREITENAU: Magnesitbez. Grazer Paläozoikum } \\
\hline $\mathrm{P}-\mathrm{Br} 1$ & Grube Horizont XII & 5249700 & -67600 & SM & 1 & -0.48 & -0.93 & -14.66 & 1.04 \\
\hline $\mathrm{P} 13$ & Grube Revier VI & 5249700 & -67600 & SM & 1 & -0.48 & -0.88 & -15.11 & 1.39 \\
\hline P16M & Grube Revier VI & 5249700 & -67600 & SM & 1 & -0.37 & -0.72 & -15.24 & 1.63 \\
\hline P16D & Grube Revier VI & 5249700 & -67600 & $\mathrm{D}$ & W & -0.04 & -0.05 & -15.30 & 1.79 \\
\hline P22a & Grube Revier V & 5249700 & -67600 & $\mathrm{D}$ & W & -0.03 & 0.05 & -15.47 & 1.98 \\
\hline P24D & Grube Revier V & 5249700 & -67600 & $\mathrm{D}$ & W & -0.09 & -0.13 & -15.55 & 2.28 \\
\hline \multicolumn{10}{|c|}{ RADENTHEIN/MILLSTÄTTER ALPE: Koralpe Wölz Deckensystem } \\
\hline R4-1 & Grube S-Feld, Sohle 7 & 5190000 & 23000 & $\mathrm{D}$ & W & -1.08 & -2.02 & -8.22 & 1.97 \\
\hline R4-3 & Grube S-Feld, Sohle 7 & 5190000 & 23000 & $\mathrm{SM}$ & 1 & -0.38 & -0.84 & -18.38 & -1.07 \\
\hline P5-1 & Grube S-Feld, Sohle 8 & 5190000 & 23000 & $\mathrm{D}$ & W & -0.67 & -1.21 & -15.54 & 0.23 \\
\hline R5-2.1 & Grube S-Feld, Sohle 8 & 5190000 & 23000 & $\mathrm{SM}$ & 1 & -0.36 & -0.70 & - & - \\
\hline R5-2.2 & Grube S-Feld, Sohle 8 & 5190000 & 23000 & SM & 1 & -0.28 & -0.49 & -16.73 & -1.26 \\
\hline R5-2.3 & Grube S-Feld, Sohle 8 & 5190000 & 23000 & SM & 1 & -0.31 & -0.58 & -18.13 & -1.57 \\
\hline \multicolumn{10}{|c|}{ KASWASSERGRABEN: Evaporitbez. Permoskyth Tir.-Nor., Bajuv. Juv. Deckensystem } \\
\hline Kas-1 & ehem. Gipsabbau & 5278300 & 102000 & SM & 1 & -0.65 & -1.27 & -7.28 & 2.02 \\
\hline Kas-2 & ehem. Gipsabbau & 5278300 & 102000 & SM & 1 & -0.74 & -1.37 & -6.29 & 2.11 \\
\hline \multicolumn{10}{|c|}{ KRAUBATH:Magnesit-Chromit-Asbestbez. Silvretta Seckau Deckensystem } \\
\hline Au5 & Augraben & 5239600 & -105400 & $\mathrm{KM}$ & 1 & -1.29 & -2.53 & - & - \\
\hline Au $31 \mathrm{M}$ & Augraben & 5239600 & -105400 & KM & 1 & -0.99 & -1.93 & -4.03 & 13.98 \\
\hline Au 33 & Augraben & 5239600 & -105400 & $\mathrm{KM}$ & 1 & -1.00 & -1.96 & -4.70 & -14.82 \\
\hline $\mathrm{Au} 45$ & Augraben & 5239600 & -105400 & $\mathrm{KM}$ & 1 & -1.32 & -2.54 & - & - \\
\hline $\mathrm{Au} 47$ & Augraben & 5239600 & -105400 & $\mathrm{KM}$ & 1 & -1.45 & -2.88 & -3.50 & -13.13 \\
\hline P-1 & Augraben & 5239600 & -105400 & $\mathrm{KM}$ & 1 & -1.32 & -2.55 & -3.06 & -13.27 \\
\hline P-LU 2D & Augraben & 5239600 & -105400 & $\mathrm{KM}$ & 1 & -1.11 & -2.15 & -5.76 & -12.05 \\
\hline
\end{tabular}




\begin{tabular}{|c|c|c|c|c|}
\hline \multicolumn{5}{|c|}{$\begin{array}{l}\text { TABELLE } 2 \\
\text { Außerösterreichisches Vergleichsmaterial }\end{array}$} \\
\hline Vorkommen & $\delta^{25} \mathrm{MgDSM}-3$ & $\delta^{26} \mathrm{MgDSM}-3$ & $\delta^{18}$ OVPDB & $\delta^{13}$ CVPDB \\
\hline \multicolumn{5}{|c|}{ Bela Stena/Serbien } \\
\hline bs-1 & -0.22 & -0.42 & 4.67 & -0.41 \\
\hline bs-2 & -0.55 & -1.07 & 5.25 & 2.67 \\
\hline bs-5 & -0.34 & -0.65 & 4.38 & 1.93 \\
\hline bs-6 & -0.49 & -0.90 & 5.34 & 2.65 \\
\hline \multicolumn{5}{|c|}{ Bushveld/Südafrika } \\
\hline Bushv-1 & -0.95 & -1.80 & 2.42 & 1.43 \\
\hline \multicolumn{5}{|c|}{ Poldasht/NW Iran } \\
\hline Pol-1 & -0.21 & -0.42 & -6.15 & -0.94 \\
\hline Pol-3 & 0.31 & 0.65 & -5.00 & 1.51 \\
\hline Pol-4 & 0.51 & 1.01 & n.b. & n.b. \\
\hline Pol-5 & -0.53 & -1.04 & -6.79 & -0.14 \\
\hline
\end{tabular}

\subsection{Kaswassergraben}

Das Vorkommen liegt in triassischen (Anis) Kalk-/Dolomitgesteinen. Der gelblich bis hellbraun gefärbte Magnesit zeigt $\mathrm{cm}$-große Kristalle und ist mit Fluorit, Bleiglanz und Zinkblende assoziiert [12]. Die Magnesitbildung erfolgte, abgeleitet aus Einschlussfluiden in den begleitenden Dolomiten, möglicherweise unter evaporitischer Beeinflussung („Salinarmagnesit") [12, 13].

- Magnesit (grobkristallin): Kas-1, Kas-2

\subsection{Kraubath}

Der kryptokristalline, reinweisse Magnesit ist an Gang- und Netzwerkstrukturen des Kraubather Serpentinitkörpers gebunden. Für seine Bildung waren Lösungs- und Anreicherungsprozesse durch deszendente $\mathrm{CO}_{2}$-reiche Wässer verantwortlich, die entlang von Störungszonen im Serpentinitkörper zirkulierten [14].

Proben:

- Rein weisser, kryptokristalliner Magnesit (Au5, Au31M, Au33, Au45, Au47, P1, LU2D).

\subsection{Außerösterreichisches Vergleichsmaterial (Tab. 2)}

\subsubsection{Bela Stena/Serbien}

Die Proben stammen aus dem stillgelegten Tagebau Bela Stena (70 km S Kraljevo/Serbien), der im von Ophiolithgesteinen umrandeten miozänen Jarandolbecken liegt. Der feinstkörnige, weiße, lakustrine Fossilien führende Magnesit weist Sedimentstrukturen, wie Lamination, intraformationelle Brekzien und Trockenrisse auf, die auf episodisches Trockenfallen des Ablagerungsraums deuten. Die Magnesitbildung erfolgte im lakustrinen Milieu durch chemische
Interaktion des Seewassers mit an Mg-angereicherten, aus dem Ophiolithbereich stammenden Wässern [15, 16].

Proben:

- Dichter, reinweisser Magnesit: bs-1, bs-2, bs-5, bs- 6 .

\subsubsection{Bushveld/Südafrika; aufgelassener Magnesitbergbau am Olifant River}

Weißer, kyptokristalliner Magnesit vom „Kraubath-Typ“ tritt in Linsen, Gängen und Netzwerken im Harzburgit des altproterozoischen Bushveld-Komplexes auf. Genetisch wird er auf Reaktionen des Gesteins mit einem $\mathrm{CO}_{2}$-reichen Fluid bei niedrigen Temperaturen zurückgeführt (Exkursionsbericht 2016, MUL, Lehrst. Geol. Lagerstättenlehre).

Probe:

- rein weisser. Kryptokristalliner Magnesit: Bushv-1.

\subsubsection{Poldasht/NW-Iran}

Der kryptokristalline, rein weiße, kreidig ausgebildete Magnesit ist rezenten und holozänen Alters. Die max. $15 \mathrm{~m}$ mächtigen Magnesitsedimente wurden/werden in evaporierenden Playabecken abgeschieden, die direkt quartären Basalten auflagern, von denen auch das für die Magnesitbildung erforderliche $\mathrm{Mg}$ bezogen wird [16, 17].

- Magnesit: Pol-1, Pol-3, Pol-4, Pol-5

\section{Ergebnisse und Diskussion}

Die untersuchten Proben sind Beispiele aus global wichtigen Typen von Magnesitlagertstätten [15, 18]. Die $\delta^{26}$ MgDsM-3-Werte (in\%o) dieser Magnesite (Tab. 1 und 2) bilden folgende für die einzelnen Lagerstättentypen charakteristischen Cluster (Abb. 4):

- Kraubath-Typ: $-2,88 \%$ bis $-1,80 \%$ 。

- Veitsch-Typ: $-1,54 \%$ o bis $-0,49 \%$ 
- Bela Stena-Typ: $-1,07 \%$ bis $-0,42 \%$

- Poldasht-Typ: $-1,04 \%$ o bis +1,01\%o.

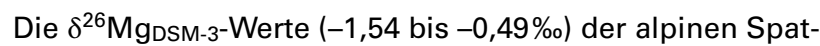
magnesite (Veitsch-Typ und Kaswassergraben) mit einer Schwankungsbreite von 1,05\% entsprechen dem durch den chinesischen Spatmagnesit vorgegebenen Rahmen [2]. Die geringe Anzahl der untersuchten Proben lässt aber offen, ob für die einzelnen Lagerstätten, die unterschiedlichen Lagerstättenbezirken angehören, signifikantere Gruppierungen der $\delta^{26} \mathrm{MgSSM}_{\mathrm{D}-3}$-Werte erwartet werden können (Abb. 1). Unklar ist auch, ob unterschiedliche Temperaturen bei der Metasomatose zu Gruppenbildungen führen können. Eventuell sind dazu aber aus der Kombination der Untersuchungen der Mg-Isotopie mit der O, C-Clumped Isotopie [19] wesentliche Erkenntnisse zu erwarten.

Generell lassen Prozesse der Metasomatose Gruppierungen der $\delta^{26} \mathrm{Mg}_{\mathrm{DSM}-3}$-Werte erwarten, die im isotopisch schweren Bereich diagnetisch gebildeter mariner Dolomite/Kalke ansetzen $[2,20]$ und darüber hinaus bis in Bereiche um $-0,49 \%$ führen (Abb. 1 und 4). Ein interessantes Phäno- men sind Mg-isotopisch schwere Dolomite, die vermutlich im Zuge der Metasomatose gebildet wurden (Abb. 5).

Kryptokristalliner Magnesit (Kraubath-, Bela Stenaund Poldasht-Typ) zeigt individuelle Gruppierungen der $\delta^{26} \mathrm{MgDSM}_{\mathrm{D}-3}$-Werte, die entweder auf prozessbezogene Fraktionierungen bei der Mg-Mobilisation aus dem Umgebungsgestein oder bei der Magnesitausfällung zurückzuführen sind. Sichtbar wird das bei den untersuchten Proben des Kraubath- und Bela Stena-Typs (Abb. 4). Beide beziehen das $\mathrm{Mg}$ für die Magnesitbildung aus Serpentinit ( $\delta^{26}$ Mg $_{\mathrm{DSM}-3}$-Werte um -0,20\%o) [21]. Der beprobte Magnesit beider Lokalitäten liegt aber in zwei deutlich getrennten Bereichen (Abb. 4). Der Magnesit aus dem Bushveldkomplex liegt durchaus im Wertebereich des Kraubath Magnesits. Für die österreichische Isotopendatenbank zu untersuchen wäre, da noch unbekannt, außerdem die MgIsotopie im Magnesitbezirk des Gföhl Deckensystems Dunkelsteiner Wald, wo der Magnesit in ultrabasischen Mantelschuppen in krustalen Gneisen situiert ist.

Die $\delta^{26}$ MgDsM-3-Werte des Bela Stena-Typs überlappen in einem kleinen Bereich mit dem Poldasht-Typ. Letzterer

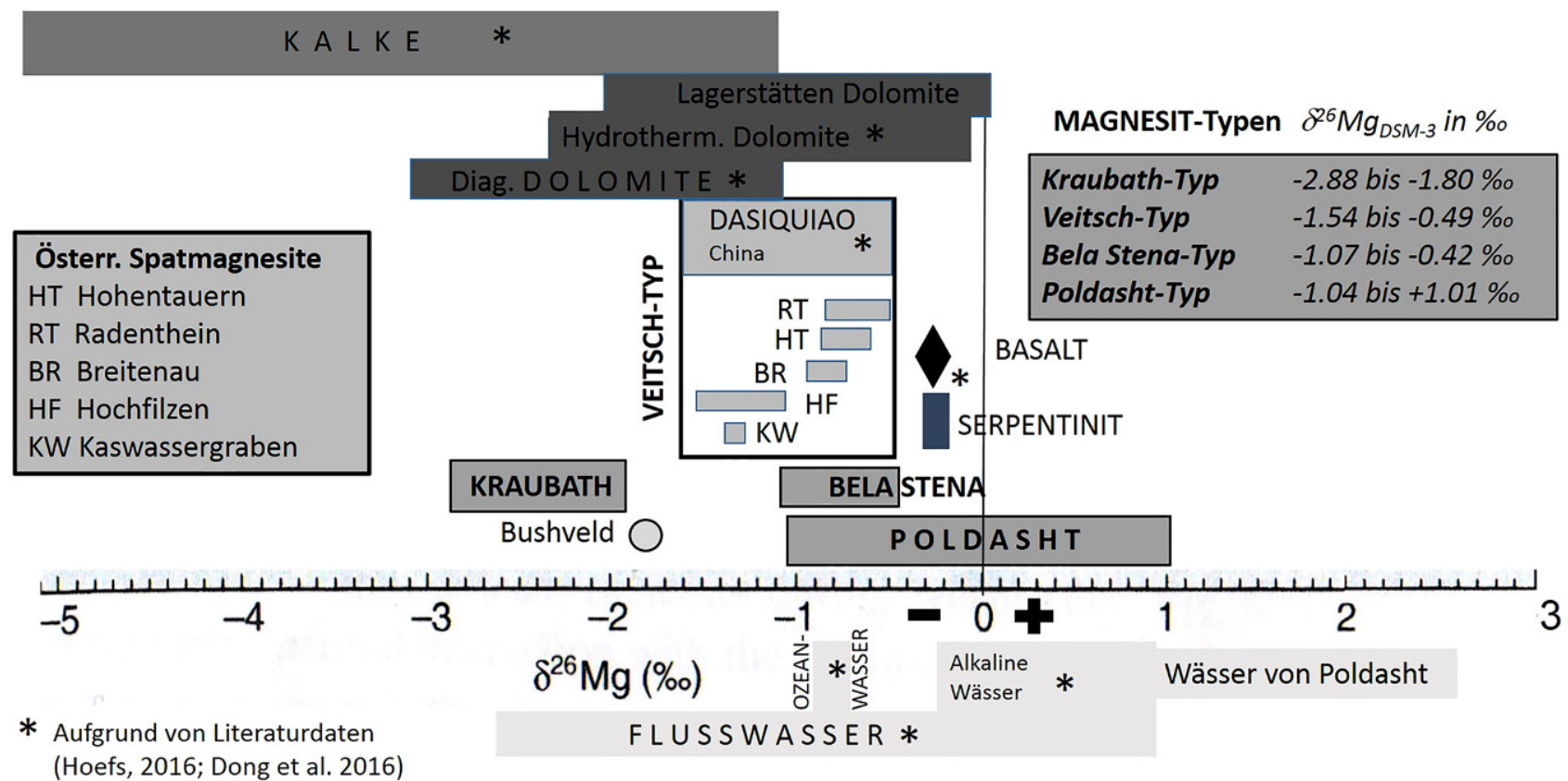

Abb. 4: $\delta^{26} \mathrm{Mg}$-Werte in Magnesit der untersuchten Proben, Typisierung der global wichtigen Magnesit-Typen und Position der untersuchten österreichischen Magnesite. Die Daten für die Wirtsgesteine sind zum Großteil der Literatur [2, 20] entnommen

Abb. 5: Im dunkelgrauen Dolomit der Breitenau (P16D) aufsprossender Spatmagnesit (P16M). a Rasterelektronenmikroskopische Aufnahme, b anpolierte Gesteinsoberfläche. Bemerkenswert ist der Mg-isotopisch schwere Dolomit im unmittelbaren Nahbereich des metasomatisch gebildeten Magnesits
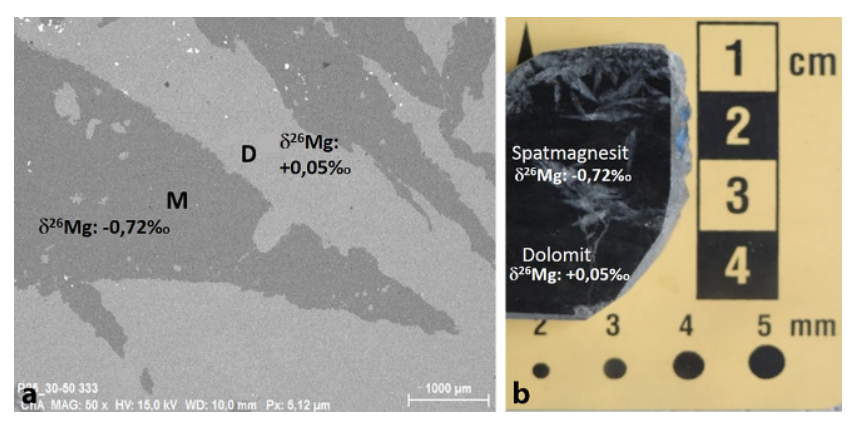
zeigt bei einer relativ großen Streuung seiner $\delta^{26} \mathrm{MgDSM}^{-3}$ Werte (von $-1,01$ bis $+1,04 \%$ o) ebenfalls nicht mehr die Isotopencharakteristik seines Mg-reichen, basaltischen Umgebungsgesteins. Dieses Ergebnis wird damit erklärt, dass es bei der fortschreitenden Evaporation hochmineralisierter alkalischer Wässer in Playa-Becken bei der Abscheidung des Magnesits zu einer Rayleigh-Isotopenfraktionierung kommen kann, bei der zuerst die leichten Mg-Isotope verbraucht und im Magnesit eingebaut werden und dadurch die schwereren Isotope im Playawasser relativ dazu angereichert werden. Folglich bildet sich im fortschreitenden Prozess der Magnesitbildung aus dem an schwereren Mglosotopen angereicherten, verbleibenden Wasser Magnesit mit einer "schwereren" Isotopie, die bis in den positiven $\delta^{26} \mathrm{MgDSM}_{\mathrm{D}-3}$-Wertebereich hineinreichen kann [17].

Die untersuchten Bespiele zeigen, dass Mg-Isotope hervorragend Einblicke in die Magnesit-Bildungsprozesse ermöglichen. Bei metasomatisch gebildetem Spatmagnesit sind aus der Kombination mit der O-, C- "Clumped “ Isotopie [19] Informationen über die Bildungstemperaturen zu erwarten. Unbekannte und innovative Aspekte eröffnen auch die mit Spatmagnesit auftretenden Mg-isotopisch schweren Dolomite (Abb. 5).

Danksagung. Die Finanzierung des Projektes erfolgte im Rahmen der GBA Forschungspartnerschaft "Grundlagenorientierte Mineralrohstoffforschung " in enger Synergie mit dem durch die Dr. Emil Suess Erbschaft der ÖAW geförderten Projekt "Magnesit im Verband mit Basalt", in dem der Poldasht-Magnesit gemeinsam mit Masoud Ovissi (Arinzamin Research Institut/Teheran) bearbeitet wurde. Für die Laboruntersuchungen standen Einrichtungen der TU-Graz (NAWI Zentrallabor für Wasser, Gesteine und Minerale) und der MU Leoben (Department für Angewandte Geowissenschaften) zur Verfügung.

Funding. Open access funding provided by Montanuniversität Leoben.

Open Access Dieser Artikel wird unter der Creative Commons Namensnennung 4.0 International Lizenz (http://creativecommons.org/licenses/ by/4.0/deed.de) veröffentlicht, welche die Nutzung, Vervielfältigung, Bearbeitung, Verbreitung und Wiedergabe in jeglichem Medium und Format erlaubt, sofern Sie den/die ursprünglichen Autor(en) und die Quelle ordnungsgemäß nennen, einen Link zur Creative Commons Lizenz beifügen und angeben, ob Änderungen vorgenommen wurden.

\section{Literatur}

1. Rantitsch, G.; Russegger, B.: Statistische Auswertung und Visualisierung der Isotopendaten österreichischer Rohstoffvorkommen, unveröff. Ber., Wien, Geowiss. Zentrum ÖAW, 2009, $17 \mathrm{~S}$

2. Dong, A.; Zhu X.K.; Li, S.Z.; Kendall, B.; Wang, Y.; Goa, Z.: Genesis of a giant Paleoproterozoic strata-bound magnesite deposit: Constraints from Mg isotopes, Precambrian Research, 281 (2016), pp 673-683

3. Wombacher, A.E.; Häusler, A.; Weyer, S.: Separation of Mg, Ca and Fe from geological reference materials for stable isotope ratio analyses by MC-ICP-MS and double-spike TIMS, Journal of Analytical Atomic Spectrometry, 24 (2009), pp 627-636
4. Weber, L. (Hrsg.): Handbuch der Lagerstätten der Erze, Industrieminerale und Energierohstoffe Österreichs, Archiv für Lagerstättenforschung, 19 (1997)

5. IRIS Online - Interaktives Rohstoffinformationssystem, https:// www.geologie.ac.at/services/webapplikationen/iris-interaktivesrohstoffinformationssystem (10.11.2018)

6. Azim-Zadeh, A.; Ebner, F.; Jiang, S.-Y: Mineralogical, geochemical, fluid inclusion and isotope study of Hohentauern/Sunk sparry magnesite deposit (Eastern Alps/Austria): implications for a metasomatic genetic model, Mineralogy and Petrology, 109 (2015), pp 555-575

7. Mavridis, A.; Mostler, H.: Zur Geologie der Umgebung des Spielberghorns mit einem Beitrag über die Magnesitvererzung, Innsbruck: Festband des Geologischen Institutes anlässlich der 300 Jahrfeier der Universität Innsbruck, 1970, S. 523-546

8. Morteani, G.: Mg-metasomatic type sparry magnesites of Entachen Alm, Hochfilzen/Bürglkopf and Spießnägel (Austria), Monograph Series on Mineral Deposits, 28 (1989), S. 105-114

9. Gallhofer, D.: Lithologische und geochemische Charakterisierung der Magnesitlagerstätte Breitenau (Grazer Paläozoikum/Ostalpen).Masterarbeit, Leoben, Montanuniv., 2010

10. Henjes-Kunst, F; Prochaska, W.; Niedermayr, A.; Sullivan, N.; Baxter, E.: Sm-Nd dating of hydrothermal carbonate formation: An example from the Breitenau magnesite deposit (Styria, Austria), Chemical geology, 387 (2014), pp 184-201

11. Tufar, W.: Magnesitlagerstätte Radenthein, in Weber, L. (Ed.): Handbuch der Lagerstätten der Erze, Industrieminerale und Energierohstoffe Österreichs, Archiv für Lagerstättenforschung, 19 (1997), S. 314

12. Prochaska, W.: Die Bedeutung der chemischen Zusammensetzung von Einschlussfluiden und laugbaren Salzen für die Genese von hydrothermalen und sedimentären Karbonatgesteinen der Ostalpen, Mitteilungen der Österreichischen Geologischen Gesellschaft, 90 (1999), S. 175-183

13. Götzinger, M.: Mineralisationen in den Gutensteiner Schichten (Anis) in Ostösterreich - Ein Überblick, Archiv für Lagerstättenforschung der Geologischen Bundesanstalt, 6 (1995), S. 183-192

14. Thalhammer, O.R.; Ebner, F; Horkel, K.; Mali, H.: Der UltramafitKomplex von Kraubath, Journal of Alpine Geology, 53 (2010), S. 137-158

15. Pohl, W.L.: Economic Geology, Principles and Practice: Metals, Minerals, Coal and Hydrocarbons - an Introduction to Formation and Sustainable Exploitation of Mineral Deposits, Oxford: Wiley-Blackwell, 2015

16. Ebner, F.; Dietzel, M.; Hippler, D.; Mali, H.: Mg-Isotopie in Magnesiten - eine Pilotstudie für den Lagerstätten-Isotopenkatalog Österreichs, unveröff. Bericht Grundlagenorientierte Mineralrohstoffforschung, Wien, GBA, 2018

17. Ebner, F.; Mali, H.; Ovissi, M.; Ghorbani, M.; Hippler, D.; Dietzel, M.: The Poldasht magnesite (W Asserbaijan, NW Iran)-a new type of magnesite deposit, Geologica Balcanica, XXI. International Congress of the CBGA, Salzburg (Austria), Sept. 10-13, 2018, Abstracts (2018), p 267

18. Ebner, F; Wilson, I.: Magnesit - globales Potenzial und geologische Lagerstättencharakteristik, BHM Berg- und Hüttenmännische Monatshefte, 151 (2006), S.164-174

19. Eiler, J.M.: „Clumped-isotope“ geochemistry-The study of naturally-occurring, multiply-substituted isotopologues, Earth and Planetary Science Letters, 262 (2007), pp. 309-327

20. Hoefs, J.: Stable Isotope Geochemistry, $7^{\text {th }}$ ed., Heidelberg/London: Springer, 2015

21. Teng, F.-Z.: Magnesium Isotope Geochemistry, Reviews in Mineralogy and Geochemistry, 82 (2017), pp 219-287 